\title{
Percutaneous embolization for a subacute gastric fistula following laparoscopic sleeve gastrectomy: a case report and literature review
}

Hung-Hsuan Yen ${ }^{1,2}$ Yu-Ting Lin², Jin-Ming Wu ${ }^{1,2}$, Kao-Lang Liü ${ }^{3}$ and Ming-Tsan Lin ${ }^{2^{*}}$

\begin{abstract}
Background: The management for subacute or chronic fistula after bariatric surgery is very complicated and with no standard protocol yet. It is also an Achilles' heel of all bariatric surgery. The aim of this case report is to describe our experience in managing this complication by percutaneous embolization, a less commonly used method.

Case presentation: A 23-year-old woman with a body mass index of $35.7 \mathrm{~kg} / \mathrm{m}^{2}$ presented with delayed gastric leak 7 days after laparoscopic sleeve gastrectomy (LSG) for weight reduction. Persistent leak was still noted under the status of nil per os, nasogastric decompression, and parenteral nutrition for 1 month; therefore, endoscopic glue injection was performed. The fistula tract did not seal off, and the size of pseudocavity enlarged after gas inflation during endoscopic intervention. Subsequently, we successfully managed this subacute gastric fistula via percutaneous fistula tract embolization (PFTE) with removal of the external drain 2 months after LSG.

Conclusions: PFTE can serve as one of the non-invasive methods to treat subacute gastric fistula after LSG. The usage of fluoroscopy-visible glue for embolization can seal the fistula tract precisely and avoid the negative impact from gas inflation during endoscopic intervention.
\end{abstract}

Keywords: Fistula, Leak, Sleeve gastrectomy, Bariatric surgery, Embolization

\section{Background}

The risk of leak after laparoscopic sleeve gastrectomy (LSG) was 2.4, and $89 \%$ of leak occurred at the proximal third of the stomach [1]. Most leaks result from the disruption of blood supply around the angle of His combined with increased intraluminal pressure and decreased gastric tube compliance after LSG [2]. Relaparoscopy or even re-laparotomy is necessary for leak happening within 24 to $48 \mathrm{~h}$ after primary bariatric surgery or anytime when uncontrolled septic signs develop

\footnotetext{
* Correspondence: linmt@ntu.edu.tw

${ }^{2}$ Department of Surgery, National Taiwan University Hospital, Taipei, Taiwan Full list of author information is available at the end of the article
}

and persist; otherwise, a non-operative management is suggested by most bariatric surgeons [3]. The management for subacute or chronic leak after bariatric surgery with fistula tract formation is more complicated, with no standard protocol yet, and also an Achilles' heel of all bariatric surgery [4-9].

\section{Case presentation}

The 23-year-old woman with the body mass index (BMI) of $35.7 \mathrm{~kg} / \mathrm{m}^{2}$ was evaluated for LSG. Her past medical history was significant for bilateral knee osteoarthritis and had taken over-the-counter pain relievers for a long time with suboptimal effects. The blood tests from her primary care physician denoted prediabetes, 
borderline hypercholesterolemia, and mild elevations of the liver function tests (glycated hemoglobin 6.4\%, total cholesterol $220 \mathrm{mg} / \mathrm{dL}$, aspartate aminotransferase $39 \mathrm{U} /$ $\mathrm{L}$, and alanine aminotransferase $64 \mathrm{U} / \mathrm{L}$ ). Preoperative workups, including contrast-enhanced computed tomography $(\mathrm{CT})$ scan and abdominal sonography, further reported a moderate fatty liver. She had also attempted to lose weight through different methods, including hypocaloric diet and exercise, for many years but without success. Therefore, the patient was indicated for and underwent LSG [10].

The LSG was performed using a 36-Fr bougie as a stent, and the staple line was oversewn to prevent postoperative leak and hemorrhage. A 7-mm silicone closed wound vacuum (CWV) drain system was placed intraoperatively along the staple line with its tip at the left esophagogastric junction (EGJ). The course of hospitalization was uneventful and the patient was discharged on postoperative day (POD) 4.

However, the patient presented with mild fever, upper abdominal pain, and purulent discharge from the CWV drain on POD7. The CT scan showed a minor leak at left EGJ (Fig. 1). After risks and benefits of surgical exploration for leak repair in this situation were provided to the patient, non-operative management with broadspectrum antibiotics, nasogastric tube decompression, and external drainage via the previously inserted CWV drain was adopted [3]. Meanwhile, considering the prolonged duration of nil per os and to provide an adequate nutritional support, total parenteral nutrition (TPN) was given based on the American Society for Parenteral and Enteral Nutrition clinical guidelines for hospitalized adult patients with obesity [11]. The nutritional weight calculation was performed as follows: "nutritional weight = ideal body weight $(\mathrm{BW})+0.25 *$ (actual BW - ideal $\mathrm{BW})$ " [12]. The estimated energy expenditure was 25 $\mathrm{kcal} / \mathrm{kg} /$ day, and the calorie composition of TPN solution was customized with $48 \%$ glucose, $18 \%$ amino acid, and $34 \%$ fat emulsion. Supplementation of multivitamins and mixtures of trace elements (including zinc, copper, manganese, chromium, and iodine) was given daily if not otherwise contraindicated. Complete blood count, serum electrolytes, liver and renal functions were monitored weekly. On POD40, endoscopic examination with n-butyl-2-cyanoacrylate (Histoacryl ${ }^{\circ}$, B. Braun) and ethiodized oil (Lipiodol ${ }^{\circ}$, Guerbet) injection in a 1:1 mixture (Fig. 2a) was performed because of the persistent minor leak with daily drainage amount $<3 \mathrm{ml}[13,14]$. But, unfortunately, an oral contrast enhanced CT scan on POD48 still showed an unresolved leak with a more enlarged pseudocavity (Fig. 2b and c).

In this situation, other alternative managements, including surgical exploration, esophageal stent, over-thescope clip (OTSC), through-the-scope clip (TTSC), and

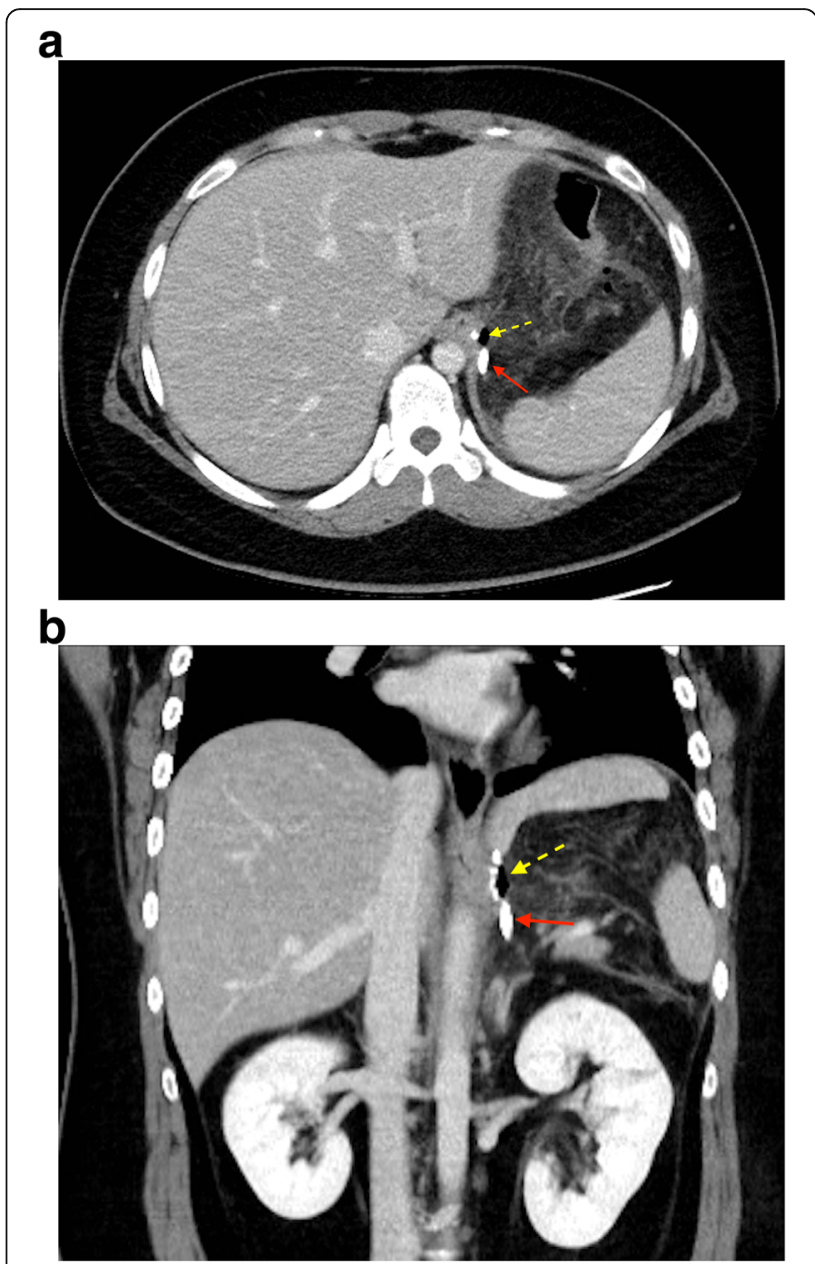

Fig. 1 CT scan, axial (a) and coronal (b) views, at POD7. The minor leak with a small air-pocket was denoted by the dashed arrow, and there was some fat-stranding nearby but no obvious fluid

accumulation. The solid arrow indicated the tip of the CWV drain placed intraoperatively. Abbreviation: POD, postoperative day; CT, computed tomography; CWV, closed wound vacuum

percutaneous fistula tract embolization (PFTE), were explained in detail and provided to the patient. After careful consideration and discussion, the patient selected and received PFTE on POD63. A guidewire was inserted through the CWV drain, and then a 40-cm 5-Fr KMP catheter $\left(\mathrm{COOK}^{\circ}\right.$ Medical) was introduced after the removal of CWV drain. After the pseudocavity and fistula tract were well illustrated under fluoroscopy, the glue $\left(33 \%\right.$, Histoacryl ${ }^{\circ}$ : Lipiodol $\left.{ }^{\circ}=1: 2\right)$ was slowly and continuously injected followed by gradual withdrawal of the catheter (Fig. 3a) [13, 14]. The follow-up oral contrast enhanced CT scan on POD68 showed the pseudocavity was sealed with glue and there was no more leak (Fig. 3b). The patient resumed oral intake since POD75 and was discharged on POD86. She had a BMI of 27 and no clinical signs of gastric leak or recurrence of the fistula in the most recent follow-up on POD183. 


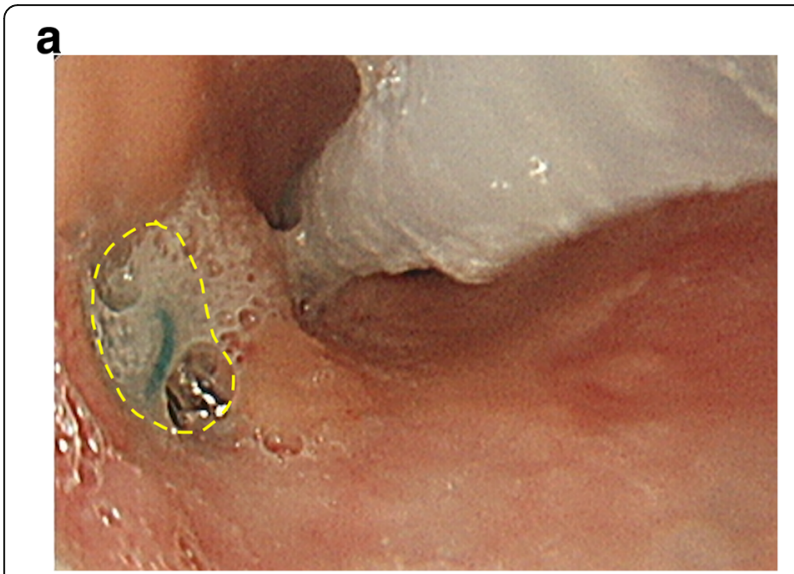

b

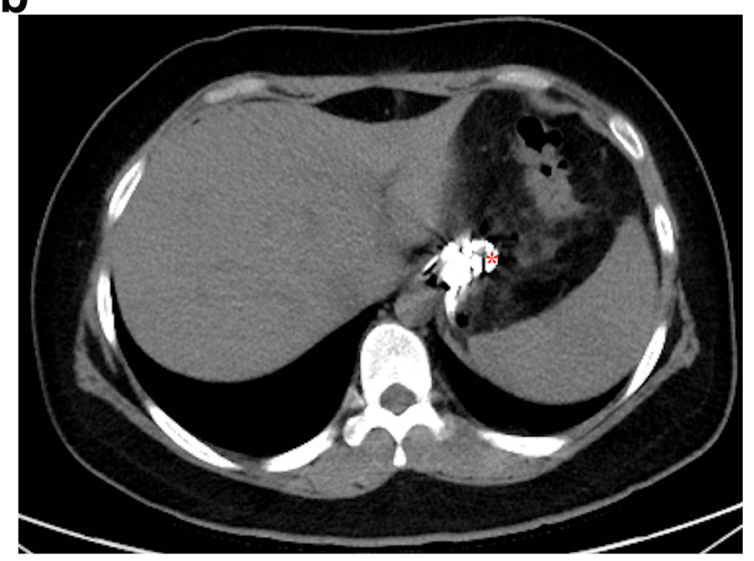

C

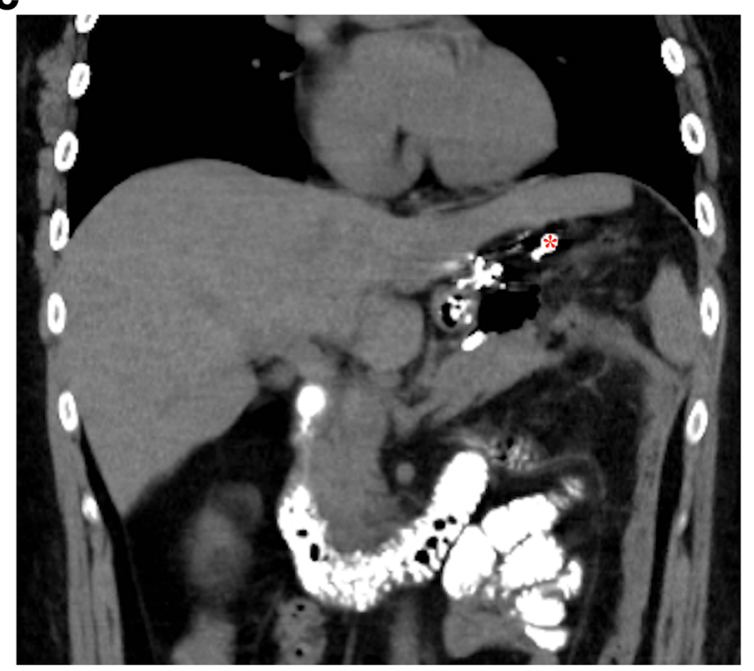

Fig. 2 Endoscopic glue injection for closure of the fistula at POD40 (a), and an oral contrast enhanced CT scan one week, at POD48, after endoscopic glue injection (b and $\mathbf{c}$ ). An about $5 \mathrm{~mm}$ fistula tract opening was found via endoscopic examination (dashed line, a), and was closed by Histoacry ${ }^{\circledR}$ and Lipiodol ${ }^{\circledR}$ injection. The following oral contrast enhanced CT scan one week later still showed a minor leak (asterisk, $\mathbf{b}$ and $\mathbf{c}$ ) and a more enlarged size of the pseudocavity. Abbreviation: POD, postoperative day; CT, computed tomography
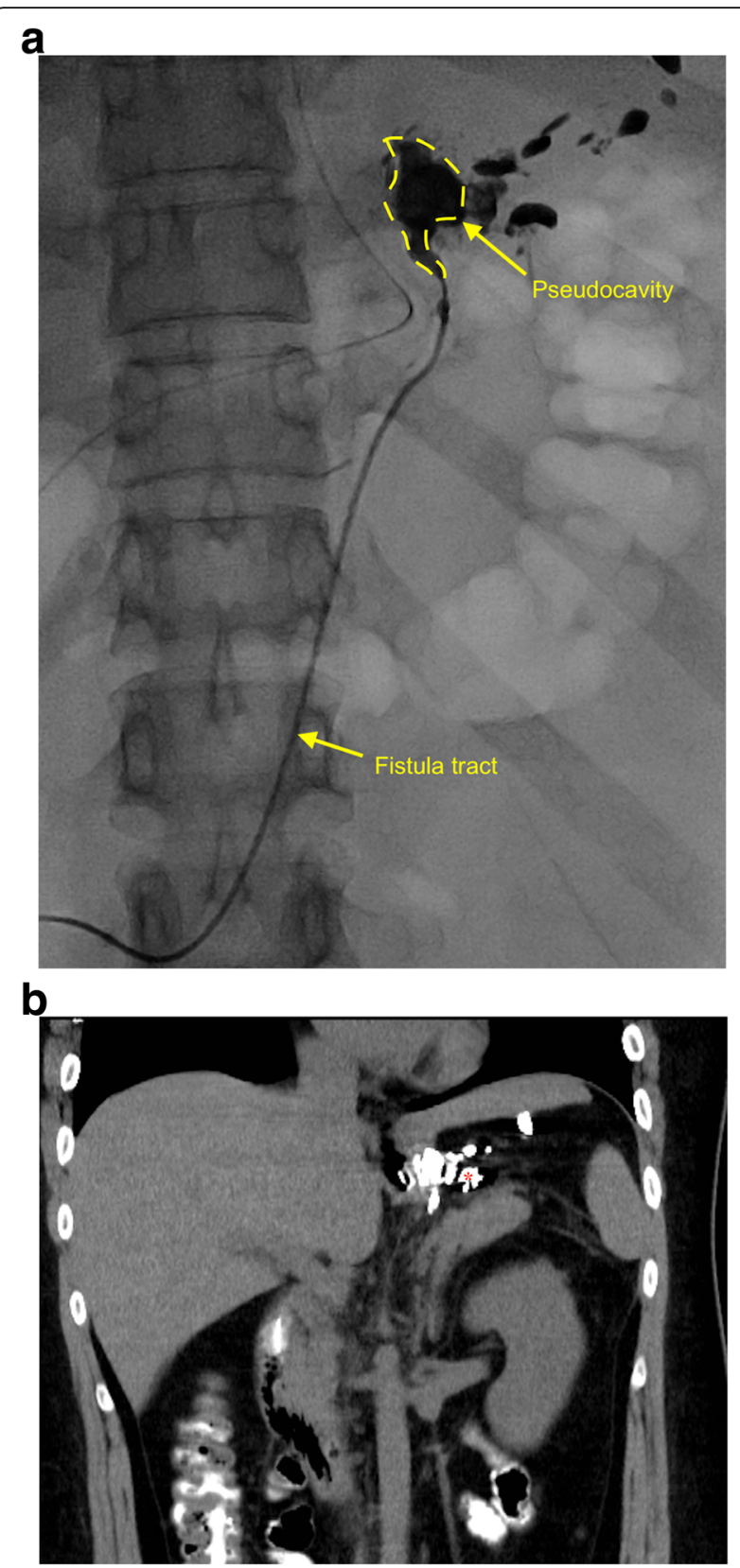

Fig. 3 Percutaneous fistula tract embolization was performed through the CWV drain insertion site at POD63 (a) and an oral contrast enhanced CT 5 days later, at POD68, showed the space of pseudocavity was filled with glue and no obvious oral contrast leak was noted (b). Abbreviation: CWV, closed wound vacuum; POD, postoperative day; $C T$, computed tomography

\section{Discussion and conclusions}

In the present report, we provided a successful experience in treating a subacute gastric fistula 2 months after LSG via PFTE with removal of the external drain following an initial failure of endoscopic glue injection 1 month after LSG. We used a fluoroscopy-visible glue to guide the embolization 
and avoided using esophagogastroduodenoscopy simultaneously to reduce the negative impact from gas inflation.

We chose PFTE to treat subacute gastric fistula for its simplicity, effectiveness, non-invasiveness, and also low cost, based on the technique and successful experiences shared by Assalia et al. [9] However, we noticed a total of 50 sessions of biological glue embolization were performed for their 23 patients with a median of 2.2 sessions per patient and $61 \%$ of all their patients required more than one application [9]. In our opinion, there are two points that would possibly improve the success rate and reduce the number of treatment session. First of all, the timing is crucial. In the early stage of gastric leak, the inflammation and infection are ongoing and the formation of pseudocavity and fistula tract has not been mature enough; therefore, at this timing, the application of glue is not able to securely seal either the fistula opening, cavity, or tract [15]. Secondly, the fibrin glue used by Assalia et al. was not illustratable on fluoroscopy, so a combined endoscopy is necessary to ensure the adequate injection of the glue. Nevertheless, gas inflation during endoscopy may worsen the pseudocavity and fistula tract as shown in our patient (Figs. 1 and 2). We added Lipiodol ${ }^{\circ}$ to the glue (Histoacryl ${ }^{\circ}$ ), and in this manner, we were able to visualize the glue injection during the whole process of embolization by fluoroscopy to ensure the precise and adequate sealing [13, 14]. Thus, we could also avoid the potentially negative effect of gas inflation during endoscopic intervention. The inclusion of $10(42 \%)$ patients with "acute" gastric fistula and the combination of endoscopic visualization (gas inflation) may explain the more treatment sessions per patient required in the study of Assalia et al. [9]

On the other hand, we did not choose endoscopic stenting or clipping, such as esophageal stent [4], OTSC [5], and TTSC [6], for two reasons. Firstly, even though esophageal stent, OTSC, and TTSC could yield a successful rate of 73 and $80 \%$ for leak after LSG respectively, there was $1 \%$ of major bleeding, $28 \%$ of stent migration, and rare complications such as esophageal rupture, tracheoesophageal fistula, or aortoesophageal fistula that could cause a catastrophic consequence $[4,5,16]$. Secondly, dealing with adverse events after elective procedure, such as bariatric surgery, is always more complicated, especially when there has been no standard method of management. The decision differs greatly between healthcare systems, costs of the instruments, patient's preferences, and patient-physician's communications. Endoscopically primary closure of the gastric fistula had also been considered for our patient with a 5-mm dehiscence; however, it was not performed concerning the high requirement of technique [8]. Recently, endoscopic internal drainage by a double-pigtail stent with prompt mobilization of the external drainage has become a promising management for hemodynamically stable patients with early leak after LSG with a successful rate of $92.8 \%$ [7]. However, whether this management is also effective for subacute or chronic gastric fistula has not been validated yet.

PFTE with fluoroscopy-visible glue materials may serve as an alternative non-invasive management for subacute gastric fistula after LSG. Nonetheless, whether this method is with high success rate requires further validation from a larger case number.

\section{Abbreviations \\ LSG: Laparoscopic sleeve gastrectomy; PFTE: Percutaneous fistula tract embolization; BMI: Body mass index; CT: Computed tomography; \\ CWV: Closed wound vacuum; EGJ: Esophagogastric junction; \\ POD: Postoperative day; TPN: Total parenteral nutrition; BW: Body weight: OTSC: Over-the-scope clip; TTSC: Through-the-scope clip}

\section{Acknowledgements}

None.

Conflict of interest

All the contributing authors declare that they have no conflicts of interest.

\section{Authors' contributions}

Conceptualization, H.-H.Y., J.-M.W., and M.-T.L.; investigation, H.-H.Y. and Y.T.L.; resources, L.-K.L. and M.-T.L.; writing —original draft preparation, H.-H.Y. and Y.-T.L.; writing - review and editing, J.-M.W., K.-L.L., and M.-T.L.;

supervision, J.-M.W., K.L.L., and M.-T.L. All authors have read and approved the final version of the manuscript.

\section{Funding}

The authors declare that they had no funding to prepare this report.

Availability of data and materials

Not applicable.

Ethics approval and consent to participate

Any accompanying images and the procedures followed were per the Declaration of Helsinki. Our internal ethics committee need not be referenced for a case report publication. The need for approval was waived.

\section{Consent for publication}

Written informed consent was obtained from the patient for publication of this case report. A copy of the written consent is available for review from the editor of this journal.

\section{Competing interests}

The authors declare that they have no competing interests.

\section{Author details}

'Department of Surgery, National Taiwan University Hospital Hsin-Chu Biomedical Park Branch, Hsinchu County, Taiwan. ${ }^{2}$ Department of Surgery, National Taiwan University Hospital, Taipei, Taiwan. ${ }^{3}$ Department of Medical Imaging, National Taiwan University Cancer Center, National Taiwan University Hospital, National Taiwan University College of Medicine, Taipei, Taiwan.

Received: 17 April 2020 Accepted: 30 September 2020

Published online: 08 October 2020

References

1. Aurora AR, Khaitan L, Saber AA. Sleeve gastrectomy and the risk of leak: a systematic analysis of 4,888 patients. Surg Endosc. 2012;26:1509-15.

2. Ferrer-Marquez M, Belda-Lozano R, Ferrer-Ayza M. Technical controversies in laparoscopic sleeve gastrectomy. Obes Surg. 2012;22:182-7.

3. Kim J, Azagury D, Eisenberg D, DeMaria E, Campos GM, American Society for $\mathrm{M}$, et al. ASMBS position statement on prevention, detection, and treatment of gastrointestinal leak after gastric bypass and sleeve 
gastrectomy, including the roles of imaging, surgical exploration, and nonoperative management. Surg Obes Relat Dis. 2015;11:739-48.

4. Okazaki O, Bernardo WM, Brunaldi VO, Junior CCC, Minata MK, de Moura DTH, et al. Efficacy and safety of stents in the treatment of fistula after bariatric surgery: a systematic review and meta-analysis. Obes Surg. 2018;28: 1788-96.

5. Yang PJ, Kuo YT, Chen CC, Wang HP. Initial experience of endoscopic clips and intrapyloric botulinum toxin injection for sleeve gastrectomy leaks. J Formos Med Assoc. 2019;118:1568-9.

6. Wang TJ, Aihara H, Thompson AC, Schulman AR, Thompson CC, Ryou M. Choosing the right through-the-scope clip: a rigorous comparison of rotatability, whip, open/close precision, and closure strength (with videos). Gastrointest Endosc. 2019;89:77-86 e1.

7. Dammaro C, Lainas P, Dumont JL, Tranchart H, Donatelli G, Dagher I. Endoscopic internal drainage coupled to prompt external drainage mobilization is an effective approach for the treatment of complicated cases of sleeve Gastrectomy. Obes Surg. 2019;29:2929-35.

8. Schweitzer M, Steele K, Mitchell M, Okolo P. Transoral endoscopic closure of gastric fistula. Surg Obes Relat Dis. 2009;5:283-4.

9. Assalia A, llivitzki A, Ofer A, Suissa A, Manassa E, Khamaysi I, et al. Management of gastric fistula complicating laparoscopic sleeve gastrectomy with biological glue in a combined percutaneous and endoscopic approach. Surg Obes Relat Dis. 2018;14:1093-8.

10. Lee WJ, Wang W. Bariatric surgery: Asia-Pacific perspective. Obes Surg. 2005; 15:751-7.

11. Choban P, Dickerson R, Malone A, Worthington P, Compher C, American Society for P, et al. A.S.P.E.N. Clinical guidelines: nutrition support of hospitalized adult patients with obesity. JPEN J Parenter Enteral Nutr. 2013; 37:7140-4.

12. Krenitsky J. Adjusted body weight, pro: evidence to support the use of adjusted body weight in calculating calorie requirements. Nutr Clin Pract. 2005;20:468-73.

13. Lee CW, Liu KL, Wang HP, Chen SJ, Tsang YM, Liu HM. Transcatheter arterial embolization of acute upper gastrointestinal tract bleeding with N-butyl-2cyanoacrylate. J Vasc Interv Radiol. 2007;18:209-16.

14. Takasawa C, Seiji K, Matsunaga K, Matsuhashi T, Ohta M, Shida S, et al. Properties of N-butyl cyanoacrylate-iodized oil mixtures for arterial embolization: in vitro and in vivo experiments. J Vasc Interv Radiol. 2012;23: 1215-21 e1.

15. Avalos-Gonzalez J, Portilla-deBuen E, Leal-Cortes CA, Orozco-Mosqueda A, Estrada-Aguilar Mdel C, Velazquez-Ramirez GA, et al. Reduction of the closure time of postoperative enterocutaneous fistulas with fibrin sealant. World J Gastroenterol. 2010;16:2793-800.

16. Donatelli G, Dhumane P, Vergeau BM, Dumont JL, Tuszynski T, Meduri B. Successful removal from the esophagus of a self-expandable metal stent that had shriveled up into a tangled ball. Endoscopy. 2013;45(Suppl 2 U(TN):E410-1

\section{Publisher's Note}

Springer Nature remains neutral with regard to jurisdictional claims in published maps and institutional affiliations.

Ready to submit your research? Choose BMC and benefit from:

- fast, convenient online submission

- thorough peer review by experienced researchers in your field

- rapid publication on acceptance

- support for research data, including large and complex data types

- gold Open Access which fosters wider collaboration and increased citations

- maximum visibility for your research: over $100 \mathrm{M}$ website views per year

At $\mathrm{BMC}$, research is always in progress.

Learn more biomedcentral.com/submissions 